\title{
CONCEPCIONES ALTERNATIVAS Y ERRORES CONCEPTUALES DE ESTUDIANTES SOBRE MODELOS ÁTOMICOS EN QUIIMICA
}

\section{ALTERNATIVE CONCEPTIONS AND MISCONCEPTIONS OF STUDENTS ABOUT ATOMIC MODELS IN CHEMISTRY}

\author{
Candelaria Tejada Tovar*, Camilo Gattas Obeid**, Ángel Villabona Ortiz***
}

\begin{abstract}
Resumen
Nuestro trabajo de investigación pretende que el estudiante descubra la trascendencia del concepto de átomo para la comprensión de la ciencia química, que en contraposición al aprendizaje incompleto, mecánico y memorístico, permita un aprendizaje significativo, de tal forma que el estudiante logre explicar la realidad macroscópica comprendiendo el mundo submicroscopico que la soporta.
\end{abstract}

En una muestra de estudiantes se realizó un diagnóstico sobre las concepciones alternativas, conocimientos previos y errores conceptuales que ellos tienen respecto al concepto de modelos atómicos, mediante la aplicación de un instrumento propuesto por las técnicas Assessmet. Este sondeo permite proporcionar información útil sobre el conocimiento de los estudiantes de determinados tópicos, además de sus habilidades en comunicar lo que saben y proporcionar una línea de base que los profesores podemos usar para tomar decisiones críticas y razonadas respecto a la didáctica y a las directrices instruccionales antes de comenzar con el proceso.

Al aplicar el instrumento se identificó una macrocategoría que corresponde a como los estudiantes expresan los modelos de átomos, identificando ocho subcategorías sobre el modo que usan para expresar estos modelos: completa gráfica y escrita, gráfica incompleta y escrita, gráfica incompleta y sin información escrita, gráfica completa y sin información escrita, solo información escrita completa, solo información escrita incompleta, mapa conceptual y no reporta información. Quedando en evidencia la influencia de las diferentes formas como enseñan los docentes y las diversas formas y estilos como aprende los estudiantes.

Palabras clave: Aprendizaje significativo, enseñanza de la química, modelos atómicos, valencia química.

* Magíster en Educación. Especialista en Química analítica Docente de planta del programa de Ingeniería Química de la Universidad de Cartagena. Investigador grupo GIPIQ inscrito en Colciencias. Email: candelariatejada@yahoo.com

** Magíster en educación Docente. Especialista en Ecología, Ambiente y Desarrollo. Email: camilogattas@gmail.com

*** Candidato a Magíster en Ingeniería Ambiental. Grupo de investigación GIPIQ, Docente de planta del programa de Ingeniería Química, Universidad de Cartagena. Email: angelvillabona@yahoo.es 


\section{Abstract}

Our research work seeks that the student discover the significance of the concept of the atom for the understanding of chemical science, which in contrast to the incomplete, mechanical and rote learning, allows meaningful learning, in such a way that the student can explain the macroscopic reality while understanding the submicroscopic world that supports it. In a sample of students, a diagnosis was applied to identify alternative conceptions, prior knowledge and misconceptions they have about the concept of atomic models, by applying an instrument proposed by Assessmet techniques. This sampling can provide useful information about the students' knowledge of certain topics, as well as their skills in communicating what they know and provides a baseline that teachers can use to make critical and reasoned decisions about teaching and instructional guidelines before beginning the process. Through the application of the instrument, a macro category was identified, which corresponds to the form students express models of atoms. Eight subcategories of the way they used to express these models were identified: complete graphic and written, incomplete graphic and written, incomplete graphic without written information, full complete graphic without written information, only complete written information, only incomplete written information, conceptual map and does not report information. It became evident the influence of the different ways teachers teach and the various forms and styles how students learn.

Key words: Meaningful learning, teaching of chemistry, atomic models, chemical valence.

\section{Introducción}

El desarrollo histórico-cultural del concepto de valencia química, se inició cuando se pensó que las partículas que componían las sustancias estaban formadas por átomos unidos, momento en el que surgió la inquietud sobre la manera en que los átomos se unían para formar compuestos (Gallego, Pérez, Uribe \& Cuéllar, 2004).

Las leyes de las proporciones definidas y múltiples han sido consideradas como la base de la construcción histórica de los conceptos de enlace químico y valencia, relacionando aquí los aportes hechos por John Dalton a principios del siglo XIX, quien propuso que los átomos tienen determinados puntos de unión; Thomas Thomson en 1813 planteó que un número de átomos de un elemento se unen con un número de átomos de determinado elemento; esto sirvió de soporte para que Eduard Frankland en 1852 propusiera el concepto de atomicidad entendido como la capacidad de combinación de los átomos de un elemento. Todo esto sirvió para que Dimitri Mendeleiev propusiera un sistema de clasificación periódica de los elementos, en donde relacionó la valencia de cada uno de ellos con su ubicación en la tabla periódica y para que Friedrich Kekulé, en 1865, propusiera estructuras de compuestos aromáticos aplicando la teoría de atomicidad, lo que más tarde se consideró como valencia química. Richard Abegg fue el primero en asociar el tér- mino de valencia química con la distribución de electrones en el átomo, afirmando en 1904 que todo átomo tiene una valencia positiva y una contra- valencia negativa. Lo anterior hace que se entienda la como valencia química, el número de electrones de la capa energética más externa de todo átomo.

Los modelos que tratan de explicar la estructura atómica se basan en esta última concepción de valencia, exceptuando el modelo de la física cuántica que a diferencia de los otros modelos, se sustenta en la ecuación de Schroedinger, que propone el concepto de orbital 0 región de máxima probabilidad de encontrar un electrón, en reemplazo del concepto de órbita, entendido como el recorrido de un electrón alrededor del núcleo, del que venían hablando los científicos anteriores. Previo a esto, Alfred Werner diferenció entre valencia primaria y secundaria, relacionando la primaria con la formación de compuestos entre elementos representativos y la secundaria para explicar la formación de compuestos complejos.

Lo anterior hace que el concepto de valencia, y consecuente explicación de los modelos atómicos, sea de carácter subjetivo y múltiple, debido a los diferentes enfoques que le han dado los autores de los textos de química, que como fuente primaria de información a través de su contenido inciden en la forma como lo enseñan los profe- 
sores, quienes realizan su propia interpretación, que a su vez repercute en la comprensión que haga el estudiante. El carácter subjetivo y múltiple, condujo a abordar el presente estudio contemplando una relación interactiva entre estudiantes y grupo investigador.

\section{Breve marco teórico}

De acuerdo con Uribe \& Gallego (2005), la enseñanza de las teorías y modelos sobre la estructura atómica de los materiales, tanto a nivel escolar como universitario, ha de basarse en el conocimiento de las concepciones alternativas que tengan los estudiantes al respecto. Los objetivos de la educación en este campo de la ciencia deben estar de acuerdo con el desarrollo cognoscitivo de los estudiantes en lo que se refiere a las etapas de educación primaria y secundaria. La enseñanza de las teorías y modelos atómicos ha de hacerse con una visión epistemológica e histórica, que permita que los estudiantes entiendan el significado de un modelo científico y lo que este representa en el desarrollo de la ciencia, para aumentar su capacidad de pensamiento crítico.

En este mismo sentido Uribe \& Gallego (2005), manifiestan que conviene dar a los estudiantes la oportunidad de discutir sobre los conceptos aprendidos y sobre cómo entendieron el desarrollo de la ciencia en este campo del saber, para desarrollar su capacidad de argumentación reflexiva. Dado el carácter abstracto del concepto de átomo y de los demás conceptos relacionados con este, han de utilizarse para su enseñanza modelos didácticos adecuados, con una secuencia lógica dentro del contexto histórico, para que los estudiantes construyan su propio modelo mental, acorde con el modelo científico.

En un estudio realizado por Martín (2001), reporta que los conceptos de elemento y átomo son comprendidos con una fuerte asociación entre sí por los estudiantes, incluso considera que existen dificultades para la comprensión de los conceptos de átomo, moléculas y compuestos. Además, algunos investigadores como Furio \& Furio (2003), han detectado que los estudiantes cometen generalizaciones erróneas en cuanto a las propiedades macroscópicas de la materia, aplicándolas al mundo microscópico.

De manera tal, que resulta importante el estudio y comprensión de la naturaleza corpuscular de la materia como antecedente ante el estudio de los modelos atómicos.

\section{Metodología}

El enfoque investigativo es de tipo cualitativo desde una perspectiva histórico-cultural debido a que se indagó sobre el desarrollo histórico-cultural del concepto valencia dada su evolución a través de la historia.

\subsection{Diseño}

La presente investigación es de tipo descriptivo, en donde la información recolectada durante la implementación de la didáctica para enseñar el concepto de valencia química, fue analizada bajo la estructura del modelo de Harry Wolcott que consta de tres partes: la descripción, el análisis y la interpretación, articulándolas de manera simultánea al presentar los resultados.

La descripción para Wolcott (1994), citado en Lucca \& Berrios (2003), contiene diez modos distintos de organización de la información. En nuestro caso, se realizó desde el denominado «focalización progresiva», en el cual se tiene en cuenta la información obtenida desde la actividad desarrollada en la primera sesión de clases (Base orientadora de la Acción), hasta la cuarta sesión (valoración de la didáctica).

Los investigadores, teniendo en cuenta los créditos académicos de la asignatura Química Inorgánica y el tiempo estipulado, en el microcurriculum, para abordar la temática de: tabla periódica, compuestos inorgánicos de los grupos representativos, y compuestos inorgánicos de los elementos de transición, se identificó que el número total de sesiones (encuentros con los estudiantes) para la recolección de la información seria de 3 horas semanales durante 4 semanas, las cuales se desarrollaron de la siguiente manera:

Se capacitó a los estudiantes con dos propósitos diferentes, el primero orientado al «como», es decir a los procesos metodológicos que el estudiante requerirá para participar en la presente investigación, y el segundo al «que» referente a los contenidos básicos relacionados con la misma. El «como» se abordó desde la teoría de la Pedagogía Conceptual de Miguel de Zubiría (2005). El docente investigador dá instrucciones a los estudiantes para la elaboración de mapas conceptuales y mapas proposicionales de manera teórica y práctica con el acompañamiento del observador participante. 


\subsection{Participantes}

Estudiantes de primer semestre de ingeniera química en la asignatura de química general, de una universidad de la Ciudad de Cartagena Colombia, quienes serán los observados en el proceso de investigación. 3 investigadores; representados en un observador participante y dos observadores no participantes

\subsection{Técnicas e instrumentos}

Las técnicas y los instrumentos a utilizar en la presente investigación se resumen en: revisión y evaluación de conceptos erróneos/prejuicios, concepciones alternativas que tienen los estudiante; mediante un Test de conceptos previos, y entrevista a los estudiantes, mediante el instrumento de entrevista abierta.

\subsection{Procedimiento}

En una muestra de estudiantes se realizó un diagnóstico sobre las concepciones alternativas, conocimientos previos y errores conceptuales que ellos tienen respecto al concepto de modelos atómicos. Mediante la aplicación de un del instrumento propuesto por las técnicas Assessmet. En cuanto al desarrollo de la didáctica se diseñaron un serie de actividades las cuales se desarrollaron tal y como se relacionan a continuación.

Para identificar las concepciones alternativas, conocimientos básicos y errores conceptuales que tienen los estudiantes sobre modelos atómicos, se realizó un sondeo en los conocimientos previos de los estudiantes puesto que no solo podía proporcionar información útil sobre el conocimiento de los estudiantes de determinados tópicos, sino también de sus habilidades en comunicar lo que saben, además proporciona una línea de base que los profesores podemos usar para tomar decisiones críticas y razonadas respecto a la didáctica y a las directrices instruccionales antes de comenzar con el proceso. Se consideró de suma importancia, que antes de empezar a orientar un concepto nuevo en el programa de estudio, los docentes debían tener en cuenta lo que los estudiantes pueden saber acerca de él, con la intención de que el estudiante, de una manera consciente reconozca que sus conocimientos pueden ser parciales, fragmentarios, simplistas, o aún erróneos.

El sondeo de conceptos previos se realizó mediante la aplicación de una pregunta abierta, de respuesta, con el fin de cumplir con lo planteado anteriormente. Se les enfatizó a los estudiantes, en que este sondeo de conceptos previos no era un examen o prueba corta a calificar, así mismo se animó a los estudiantes a dar respuestas razonadas que pudieran ayudarnos en el proceso de toma de decisiones con respecto a las instrucciones a seguir en el proceso. Igualmente se les explicó que la información que surge a partir de esta prueba de conceptos previos, es de gran utilidad para aclarar dudas, reforzar conceptos débiles 0 corregir errores conceptuales, así como de base para contextualizar la instrucción sobre el nuevo tema, en nuestro caso el concepto de valencia química.

Para convertir los datos recolectados en información útil, se analizaron las respuestas dadas por los estudiantes y se organizaron categorías (macrocategorías y subcategorías), posteriormente se identificaron los errores conceptuales, conocimientos básicos no relevantes y conocimiento básico relevante que tenían los estudiantes.

\section{Resultados y discusión}

Con respecto a la pregunta, donde se le indagaba a los estudiantes que compararan los modelos atómicos de Dalton, Thomson y Rutherford, se identifica una macrocategoría que corresponde a la forma como los estudiantes expresan los modelos de átomos y se identificaron ocho subcategorías que se describen a continuación, esta pregunta la consideramos pertinente dado que la didáctica se desarrolló teniendo en cuenta la génesis y desarrollo epistemológico en el que se debe enseñar todo concepto en Química; de igual forma se procedió para el análisis de todas las preguntas:

Diez estudiantes de cuarenta representan gráficamente y dan una información escrita, un ejemplo de esto es el estudiante $N^{\circ} 6$, tal como se muestra en la figura 1.

Esto da una muestra de que probablemente en algunos casos los docentes se apoyan para dar su instrucción con ayudas de tipo gráfico para explicar los diferentes modelos de átomo que han existido a lo largo de la historia, y que los estudiantes los han aprendido de memoria, pues en el caso del estudiante que estamos citando reproduce las representaciones tal y como aparecen en algunos textos de química general. Nos parece interesante resaltar que fue el único estudiante que hizo mención a algo de histórico y epistemológico, cuando afirma que el modelo de Dalton recoge el atomismo filo- 


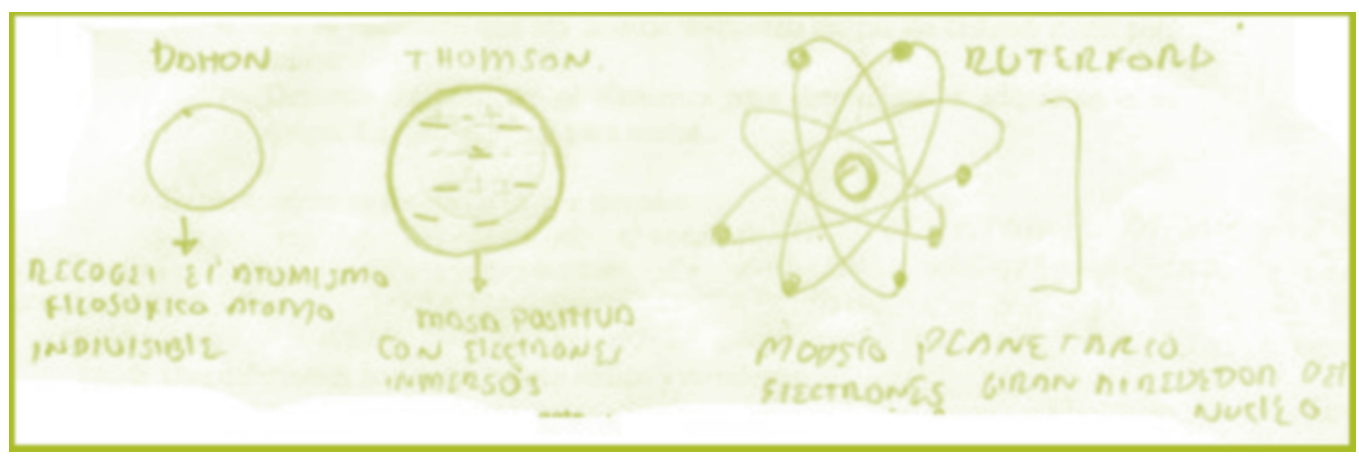

Figura 1. Representación gráfica de modelos atómicos por el estudiante $\mathrm{N}^{\circ} 6$

sófico, también por la secuencia en que el estudiante realiza sus representaciones, observamos que están organizados de tal manera que coinciden con su orden de desarrollo histórico, esto en el fondo consideramos que tiene más trascendencia para el estudiante, por lo cual quisimos ampliar su respuesta y procedimos a llamarlo y entrevistarlo.

Con respecto a lo anterior, después de hablar con el estudiante se encuentra que este tiene claro que el conocimiento que se tiene actualmente sobre el modelo de átomo, ha sido el resultado de diferentes aportes a lo largo de la historia, tal como lo afirma en la siguiente respuesta «la evolución histórica de las teorías atómicas todas tienen el modelo actual, todos tienen un pequeño aporte, ósea todos fueron necesarios en su momento para la llegada, es como decir que actualmente todos esos modelos están vigentes, al menos para decir que ese no era, si me explico». En este mismo sentido es probable que el estudiante quiera dar a entender que aunque un modelo no esté vigente es pertinente tenerlo en cuenta, denotándose que considera necesario abordar todos los aportes realizados referentes al tema en cuestión a lo largo de la historia.
Dos estudiantes representan gráficamente pero en forma incompleta y dan una información escrita, un ejemplo de esto es el estudiante $N^{\circ} 10$, Figura 2.

En este estudiante a diferencia del anterior se observa que el orden en que expones los modelos, no guarda ninguna secuencia respecto a su aparición en la historia, el hecho de que este estudiante realice una representación gráfica de algunos modelos de átomo puede obedecer a que en la instrucción de estos conceptos no se hizo mucho énfasis en la parte gráfica del modelo 0 que al estudiante no se le facilita apropiarse de la información de tipo gráfico, o que no represento una información significativa para él.

Tres estudiantes Hacen una representación gráfica incompleta y sin información escrita, un ejemplo de esto es el estudiante $\mathrm{N}^{\circ} 12$, Figura 3.

Este estudiante parece no tener claro los modelos de átomo por los que se le está preguntando, pues realiza una representación gráfica incompleta y no expresa de manera escrita algunos de sus fundamentos teóricos, esto refleja que probablemente el estudiante tiene un conocimiento

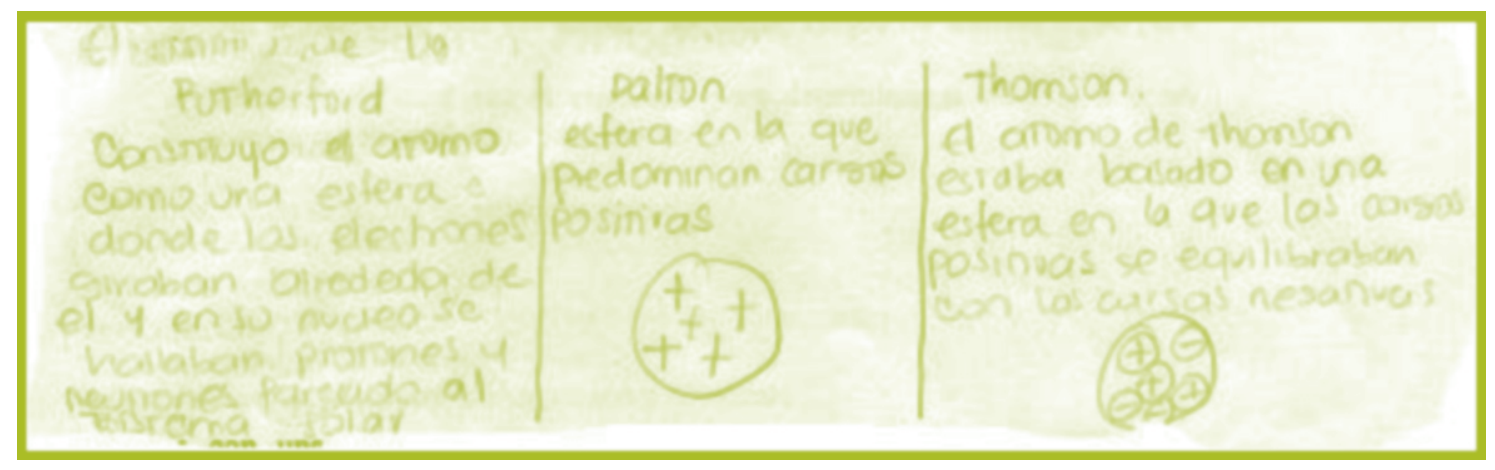

Figura 2. Representación gráfica de modelos atómicos por el estudiante N10 


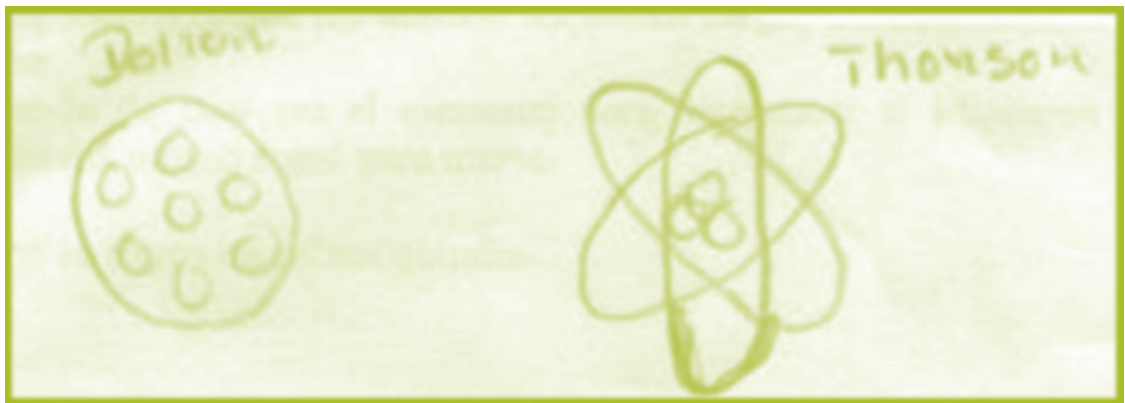

Figura 3. Representación gráfica de modelos atómicos por el estudiante N12

muy incipiente sobre el tema, lo que se puede deber a que en su momento el docente explicó de manera superficial esta temática, sin profundizar, o que el estudiante no estuviera lo suficientemente motivado respecto a este tema, como para apropiarse de él de manera significativa.

Siete estudiantes realizan una representación gráfica completa, un ejemplo de esto es el estudiante $N^{\circ} 19$, Figura 4.

Los estudiantes de esta subcategoría también muestran en su representación una secuencia histórica de los diferentes modelos de átomo por los que se le preguntó; en este caso puede ser que a los estudiantes se les facilite más el aprendizaje de manera visual es decir gráficamente o que el docente en su momento hizo énfa- sis en su instrucción en este tipo de representaciones, sin dedicarle suficiente tiempo a la instrucción teórica, o también porque el estudiante no tiene claro los conceptos, y prefiere no complicarse al escribir cosas de las cuales no está seguro, o no son de su pleno dominio.

Nueve estudiantes presentan solo información escrita, un ejemplo de esto es el estudiante $N^{\circ} 30$, Figura 5.

Este estudiante lo que escribe no lo presenta de manera secuencial, con respecto a su aparición en la historia, quizás los docentes no fueron enfáticos en esas precisiones al momento de la orientación, restándole importancia, lo que imposibilita un aprendizaje genuino; o el estudiante es quien ha pasado por alto este detalle, al no percibir

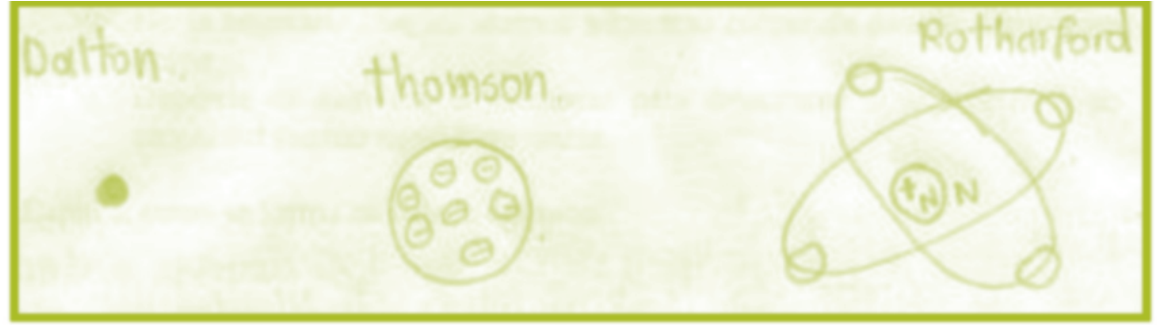

Figura 4. Representación gráfica de modelos atómicos por el estudiante №19

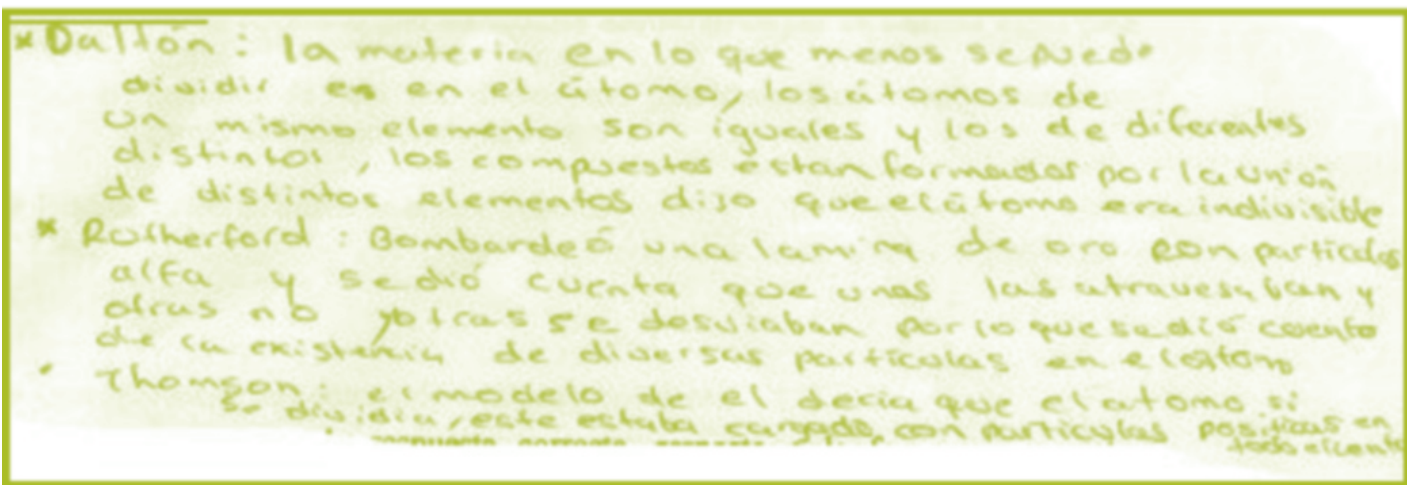

Figura 5. Representación gráfica de modelos atómicos por el estudiante $\mathrm{N}^{\circ} 30$ 
la importancia de este hecho. El estudiante al no utilizar representaciones gráficas de los modelos, da muestra de que probablemente el docente en su instrucción no enfatizó en dichos modelos de manera gráfica o que a él se le dificulta realizar este tipo de representaciones.

Cuatro estudiantes presentan información escrita incompleta, un ejemplo de esto es el estudiante $N^{\circ} 23$, Figura 6.

Este estudiante tal vez se le dificulta realizar una representación gráfica, o puede ser que no tenga claridad respecto a los modelos, aun cuando expresa de manera incompleta algunos de sus fundamentos teóricos, se nota que no tiene un conocimiento completo respecto a lo que se le estaba preguntando.

Un estudiante realizó un mapa conceptual sin dibujar los modelos, como ejemplo el estudiante $N^{\circ} 40$, Figura 7.

Este estudiante da muestra de tener una buena capacidad de síntesis y presenta de manera escrita y bien organizada la información respecto a los modelos de átomo, también hace una presentación de los modelos con una secuencia lógica respecto a su desarrollo a lo largo de la historia.

Por último con respecto a la primera pregunta se pudo identificar que cuatro estudiantes no presentaron ningún tipo de información.
Los estudiantes de esta subcategoría parece ser que no tienen claro los conceptos respecto a los modelos de átomo o puede ser que en la instrucción que les dieron sus profesores no hicieron énfasis en la importancia de conocer el desarrollo histórico del concepto de átomo, o los estudiantes simplemente recibieron estos conceptos como simple información y no la convirtieron en conocimiento importante para ellos, por tal motivo cuando se les pregunto al respecto no recordaron con claridad y prefirieron no escribir nada, o tal vez puede ser que no tengan ningún conocimiento al respecto.

En general, podemos señalar después de hacer una revisión de las respuestas dadas a esta pregunta, que la información que suministran es puntual y sin relación, desconociendo que es un concepto en construcción, el hecho de tener esta información no es garantía para que asocien el comportamiento de la materia que experimentan en su cotidianidad con la estructura atómica. Esto se puede generalizar para los estudiantes que solo dieron una información escrita y para aquellos que solo representaron gráficamente, cuando se les indagó sobre los diferentes modelos de átomo.

\section{Conclusiones}

Se identificó como macro categoría: La forma de expresar los estudiantes los modelos de átomos, y como

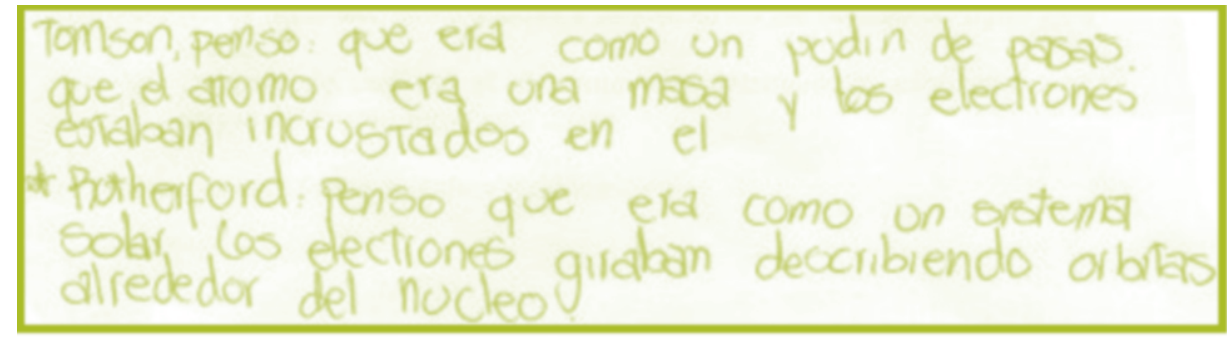

Figura 6. Representación gráfica de modelos atómicos por el estudiante N²3.

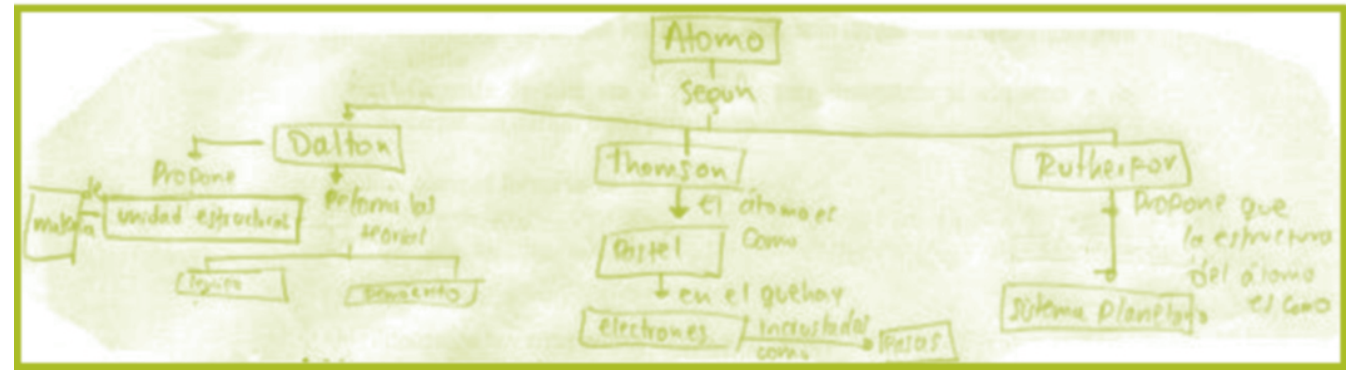

Figura 7. Representación gráfica de modelos atómicos por el estudiante Nㄴ. 


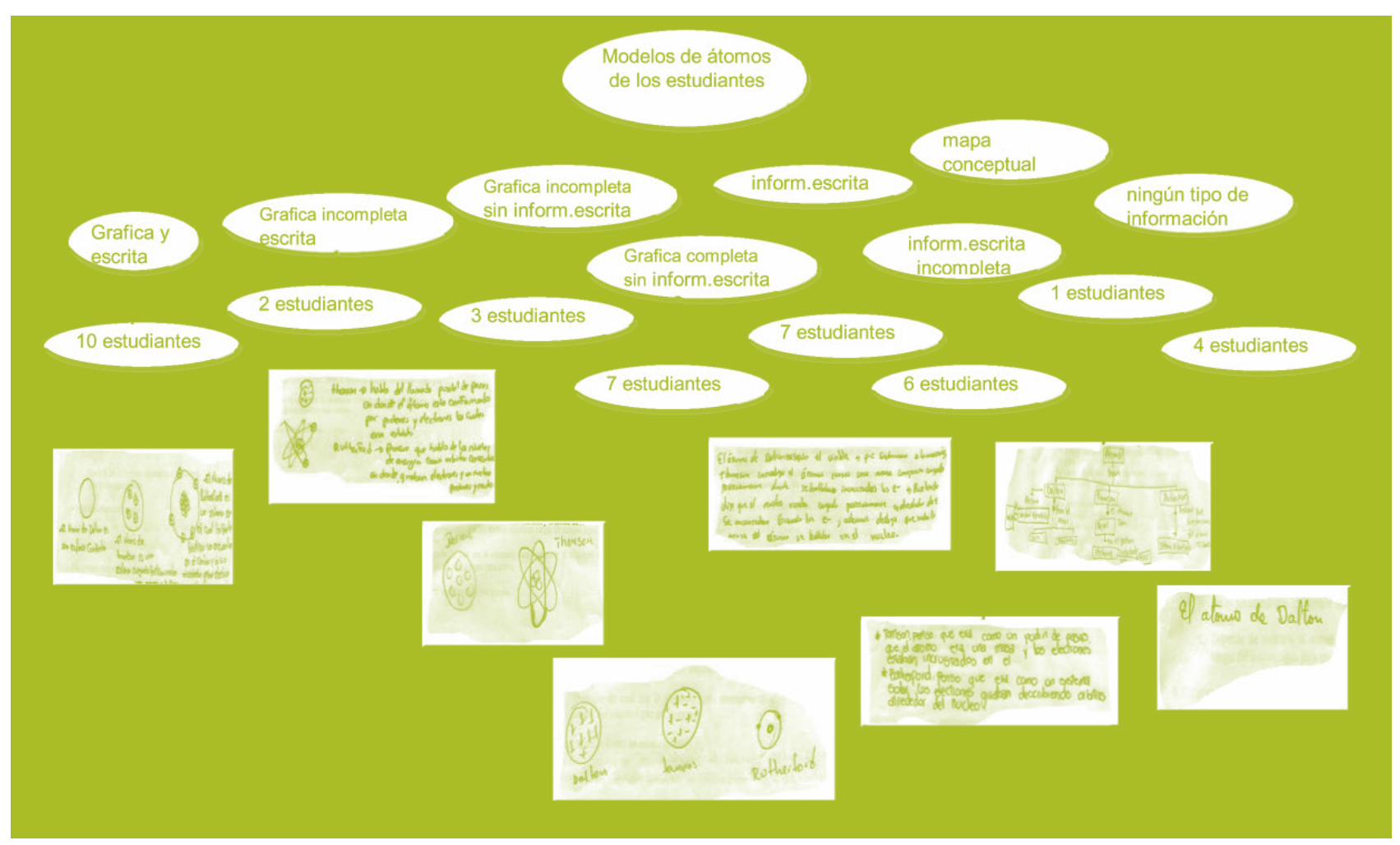

Figura 8. Mapa conceptual sobre modelos atómicos de los estudiantes

subcategorías: se identificaron 8 formas de expresión por parte de los estudiantes. En forma completa gráfica y escrita, en forma gráfica incompleta y escrita, en forma gráfica incompleta y sin información escrita, en forma gráfica completa y sin información escrita, solo información escrita completa, solo información escrita incompleta, mapa conceptual y no reporta información, lo que pone en evidencia los diferentes tipos de aprendizajes de los estudiantes y probablemente el efecto que tienen los diferentes estilos de enseñanza de los docentes. En el mapa conceptual que se muestra en la figura 8 , se sintetizan la relación entre la macrocategoría y las subcategorías que emergieron del análisis de la información que suministraron los estudiantes respecto a la pregunta sobre modelos atómicos.

\section{Referencias bibliográficas}

De Zubiría, M. Pedagogías del siglo XXI: Mentefactos I: Fundación Internacional de Pedagogía Conceptual Alberto Merani. Fondo de publicaciones Fundación Alberto Merani,2005.
Furio. C, \& Furio. C. Dificultades conceptuales y epistemológicas en el aprendizaje de los procesos químicos. Revista de Aniversario. Educación química. 11(3)(2003): 300.

Gallego. R, Pérez. R, Uribe. M, \& Cuéllar. L. El Concepto de Valencia: Su construcción histórica y epistemológica y la importancia de su inclusión en la enseñanza. Ciencia y educación. 10(3)(2004):571-583.

Lucca. N, \& Berrios. R. Investigación Cualitativa en Educación y Ciencias Sociales. Publicaciones Puertorriqueñas. Puerto rico. 2003.

Martín del Pozo, R. Prospective teachers ideas about the relationships between concepts describing the composition of matter. International Journal Science Education: 23(4)(2001):353-371.

Uribe. M.V, \& Gallego. R. Enseñanza de los modelos atómicos en programas de ingeniería. Educación y Educadores. 8(2005):67-76. 